\title{
Mejoras del Aprendizaje y del Rendimiento de Grupos Reducidos de Estudiantes en una Asignatura de Proyectos de Ingeniería
}

\author{
F.T. Bogumil y F. Abad \\ Universidad Politécnica de Cataluña, E.T.S.I.I. de Terrassa, Departamento de Proyectos de \\ Ingeniería, c/ Colon 1, Apdo. 577, 08222 Terrassa-España
}

\section{Resumen}

Se presentan las características, resultados teóricos y conclusiones finales de una experiencia para la mejora del aprendizaje y la evaluación del rendimiento de un grupo reducido de estudiantes de la asignatura de proyectos de ingeniería de la Escuela Técnica Superior de Ingenieros Industriales de Terrassa, España. La metodología empleada consideró la aplicación de un cuestionario mediante el cual se valoraron los siguientes aspectos: trabajo individual, trabajo en grupo, preparación de presentaciones orales. Se realizaron valoraciones durante el curso académico permitiendo detectar a tiempo tos alumnos con bajo rendimiento y posibilitando su reintegración a la dinámica del grupo. Los resultados obtenidos demuestran que las nuevas técnicas de evaluación del rendimiento permiten conseguir los objetivos de motivación por el curso, cohesión del grupo y aprendizaje de las materias.

\section{Improvement of Learning and Teaching Efficiency of Reduced Groups Of Students in an Engineering Project Course}

\begin{abstract}
The characteristics, theoretical results and final conclusions of an experience for the improvement of the learning process and of its evaluation of a reduced group of students of a university course, is presented in this article. The experiment was done with an engineering project course at the Technical University School of Industrial Engineers of Terrassa, Spain. The methodology considered the application of a questionnaire to evaluate the following aspects: individual work, group work, and preparation of oral presentations. During the academic course, several evaluations were done which allowed detecting students with low efficiency and making the necessary corrections to integrate them into the dynamics of the group. The results demonstrate that the new evaluation method allowed to achieve the objectives of motivation for the course, cohesion of the group and subject learning.
\end{abstract}

Keywords: teaching methods, evaluation, teaching-learning, engineering project courses 


\section{INTRODUCCION}

Los aspectos básicos de la evaluación del rendimiento se pueden dividir según Nisbet y Shuckmiry (1987) en: concepto, finalidad y aplicación. Dentro del Concepto, se tiene que las técnicas de evaluación del rendimiento engloban un conjunto de aportaciones científicas y técnicas que permiten, de una manera rigurosa, evaluar sin subjetividad ni errores de método. Como explica Anguera (1985), las técnicas de evaluación son útiles para las organizaciones en la consecución racional y endenté de sus objetivos. Su ámbito de aplicación es amplio y altamente diferenciado. Por esta razón, y para evitar una excesiva generalización, este trabajo estará centrado en organizaciones del tipo Universidades y Centros de Educación. En particular, el sistema objeto de estudio constará de un profesor y un grupo reducido de estudiantes universitarios de la asignatura de proyectos. Según Apple y King (1983), existen múltiples técnicas de evaluación en función del tipo de organización. Por ello, se buscará una solución que contenga: i) elementos integrados de técnicas y conocimientos ya conocidos y ii) elementos críticos que permitan adaptar estas técnicas a la realidad de la organización objeto de estudio.

La finalidad de la evaluación en el ámbito educativo tiene diversos objetivos encaminados al aprendizaje de la materia impartida entre ellos, motivar y alentar un mejor conocimiento de la asignatura, fomentar el espíritu de trabajo individual o en grupo, etc. Parece adecuado que el sistema de valoración escogido permita discretizar cada uno de estos conceptos susceptibles de valoración. A la vez, con la finalidad de establecer un criterio de comparación, el sistema debiera proporcionar una única valoración final. Bogumil (1992), ha demostrado que en un sistema profesor tutor-grupo reducido de alumnos, la evaluación del rendimiento ha sido tradicionalmente aceptada como necesaria, aunque el método de evaluación dependiera del criterio personal del profesor. Se hace necesaria una norma rigurosa y objetiva que proporcione una base común al global del colectivo evaluado, a partir de la cual comparar y evaluar. Al plantearse la evaluación de un modo amplio, surge el problema de que evaluar. Se ha mostrado en literatura (Huse y Bowditch, 1989; Olszewski, 1975) que en función del tipo de organización o de la finalidad de la evaluación, se puede evaluar: a) resultados o desempeños y b) criterios simples, compuestos o múltiples.

a) Resultados o desempeños: en el caso estudiado, el resultado sería la valoración de la tarea asignada al alumno. El desempeño incluiría todas las actividades que ha realizado el alumno para concluir la tarea. Una valoración correcta debiera incluir ambas perspectivas.

b) Criterios simples, compuestos o múltiples; cada criterio hace referencia al número de mediciones a realizar de cada alumno. El simple es el aplicado tradicionalmente, y consiste en tomar una sola medida que proporcione una valoración global del estudiante El compuesto tiene en cuenta en grupo de variables comunes a todos los evaluados. La combinación de estas mediciones proporciona una valoración individualizada que permite comparar. Los criterios múltiples de valoración agrupan diversos sistemas de medir el rendimiento. Cada uno proporciona una valoración individual de diferentes aspectos del rendimiento del evaluado.

En la aplicación de las técnicas de evaluación, también se ha mostrado en la literatura (Crum, 1978; Beard, 1974; Bleger, 1980) que para que un sistema de evaluación proporcione los resultados deseados deberá contener algunos requisitos previos: i) Aceptación de la evaluación como una necesidad fundamental de la organización. El profesor deberá ser consciente de que evaluar, forma parte intrínseca del proceso de aprendizaje; ii) Conciencia clara de la utilidad de las técnicas de evaluación aplicadas, por parte, en este caso, del profesor; iii) Clima favorable y cultura de participación. El colectivo evaluado - estudiantes - deberá aceptar tanto la evaluación en sí, como la técnica escogida para realizarla. El evaluador -profesor- deberá garantizar, formal o informalmente, rigor, objetividad y profesionalismo. Asimismo, las consecuencias y finalidad de la evaluación, deberán ser conocidas y estipuladas de antemano; iv) Estructura de tareas claramente definida y objetivos finales prefijados.

Los resultados de la evaluación pueden perder fiabilidad si las tareas objeto de evaluación cambian en el curso de la misma. El estudiante deberá saber que debe hacer y como se espera que lo haga. 
Otro aspecto importante y que se deberá decidir con el consenso de todo el grupo, es la elección de quien ha de evaluar. Según el caso podrá escoger entre: a) El profesor en general es la persona más indicada por su mayor capacidad de emitir juicios fiables. Los errores de percepción sólo se podrán evitar con una actitud que facilite la relación estrecha profesor-alumno; b) Los alumnos: es una opción que ha evidenciado ser válida como sistema de evaluación auxiliar. Los datos pueden estar distorsionados por efectos de amistades, recelos, etc.; y c) Auto-evaluación: esta opción es aconsejable si se busca la motivación y desarrollo personal. Para ser eficaz deberá formar parte de un sistema múltiple de evaluación. Una vez cumplidos cada uno de los requisitos enumerados, el siguiente paso consiste en escoger la técnica de evaluación más indicada.

\section{LA EVALUACIÓN EN EL GRUPO PLASTEC}

La descripción del curso, del grupo y la confección del cuestionario son presentadas en esta sección. Se analiza también los informes de valoración y el informe de evaluación de los alumnos y se discute los puntos que motivaron comentarios explícitos por parte de los alumnos de otros grupos.

\section{Descripción del curso}

El curso se impartió en la Escuela Técnica Superior de Ingenieros Industriales de Terrassa y su objetivo fue llevar a cabo el proyecto asignado al grupo reducido de estudiantes, a presentar a final de curso. Para realizar un seguimiento del trabajo de cada grupo reducido de estudiantes de $5^{\circ}$ curso, se realizaron tres presentaciones a lo largo del curso. Estas fueron públicas y cada estudiante matriculado debía asistir a dos de ellas, mas la correspondiente a su grupo, por cada tumo de presentaciones. La participación en las presentaciones por parte de los alumnos asistentes era activa. Se proporcionaba a los estudiantes un cuestionario en el que valoraban los aspectos positivos y negativos del grupo que realizaba la presentación. Estas valoraciones de los estudiantes, eran tomadas en cuenta por parte de los profesores. El resultado de esta valoración múltiple se presenta dividida en los siguientes conceptos: contenido, expresión, dinámica y medios.

\section{Descripción del grupo}

El grupo objeto de estudio era de dieciséis estudiantes. El trabajo se organizó en subgrupos según las áreas de trabajo y en función de la especialidad. Se eligió un jefe de grupo de estudiantes para coordinar las actividades de cada uno de los subgrupos y actuar como nexo entre profesor y alumnos. El grupo se reunía semanalmente para decidir los trabajos a realizar, comunicar propuestas e ideas, planificar el trabajo, preparar presentaciones. Para realizar un seguimiento del trabajo de cada uno de los subgrupos y, a propuesta del profesor, se comunicó a los estudiantes que se llevaría a cabo una valoración individual de cada uno de ellos después de cada presentación. Además del seguimiento por parte del profesor, se pretendía motivar a los estudiantes a mejorar las valoraciones en caso de ser estas negativas o a mantener una línea de trabajo en caso de ser positivas. La realimentación era por tanto la segunda finalidad de la valoración.

Los estudiantes fueron informados de que la valoración la realizaría el profesor en coordinación con el jefe de grupo. Después de considerar diversas opciones, se concluyó que el sistema de valoración más adecuado dada la dificultad de valorar objetivamente mediante el sistema tradicional, era el de criterio compuesto. Se valorarían tanto resultados como desempeños. Los primeros comprenderían el trabajo realizado en clase y en las presentaciones. Como desempeño se entiende todos aquellos conceptos de más difícil valoración como la actitud frente al trabajo, iniciativa,..., pero que no por ello deben ser excluidos. El no considerar este tipo de conceptos no cuantificables, es un error en que se incurre frecuentemente. Si la valoración cumple los criterios enumerados en la sección anterior, tendrá absoluta validez. Se comunicó a los estudiantes el tipo y la finalidad de la valoración, siendo ambas aceptadas por el grupo. 


\section{Confección del cuestionario}

En primer lugar se decidieron las variables susceptibles de valoración. Las variables escogidas fueron: 1) Trabajo individual; 2) Trabajo en clase; 3) Presentación preparación; y 4) Presentación.

Definidas las variables fundamentales, se escogieron los aspectos a tener en cuenta de cada una. Se intentaron incluir aspectos cuantificables como la cantidad o cualidad del trabajo realizado y aspectos menos definidos como la actitud frente al trabajo o la iniciativa para proponer ideas nuevas. Añadiendo estos conceptos a los anteriores, se obtuvo la Tabla 1. En la tabla, Inic. $=$ Iniciativa en clase, Act.= Actitud en clase, T.P.= Tiempo previsto, T.E.= Tiempo extra, y Inf.=Informe profesores

Tabla 1: Hoja de valoraciones. Conceptos

\begin{tabular}{|c|c|c|c|c|c|}
\hline $\begin{array}{c}\text { Trabajo } \\
\text { individual }\end{array}$ & $\begin{array}{c}\text { Trabajo en } \\
\text { clase }\end{array}$ & \multicolumn{2}{|c|}{ Preparación presentación } & \multicolumn{3}{|c|}{ Presentación } \\
\hline \multicolumn{2}{|c|}{ Participación } & & T.P & T.E & Inf. \\
\hline Cantidad & Calidad & & & & \\
\hline
\end{tabular}

El siguiente paso fue definir los niveles. En la elección se intentó evitar la precisión excesiva que supone la utilización de números que dificulta la labor del evaluador. Los niveles escogidos fueron: Muy Bien (MB), Bien (B), Regular (R), Suficiente (S), Insuficiente (I). Definidos los instrumentos de evaluación, quedaba únicamente organizar el seguimiento del trabajo de los estudiantes. Las fuentes utilizadas fueron:

i) El trabajo realizado por cada estudiante tanto el asignado previamente, como el producto de iniciativas o propuestas.

ii) Los datos referentes a la actitud y participación de los estudiantes en clase. Estos datos se ponían en común entre profesor y director de grupo.

iii) El trabajo destinado a preparar las presentaciones. Se consideró a la parte del trabajo destinado a proyecto porque el resultado era cuantificable en términos de transparencias, esquemas, resúmenes etc., no incluidos en el proyecto final.

iv) Los informes presentados por los estudiantes valorando otras presentaciones.

A continuación se comentan algunas conclusiones extraídas de dos valoraciones realizadas.

Tabla 2: Valoraciones de la primera presentación

\begin{tabular}{|c|c|c|c|c|c|c|c|c|c|c|c|c|c|c|c|}
\hline \multirow{2}{*}{\multicolumn{2}{|c|}{ GRUPO:92-7 }} & & & & & & & \multirow[t]{2}{*}{ PERIODO } & \multicolumn{4}{|c|}{ DESDE 01/10/1992 } & & & \\
\hline & & & & & & & & & \multicolumn{4}{|c|}{ HASTA $17 / 02 / 1992$} & & & \\
\hline \multirow{3}{*}{ ALUMNO } & \multicolumn{4}{|c|}{ TRABAJO INDIVIDUAL } & \multicolumn{4}{|c|}{ TRABAJO EN CLASE } & \multicolumn{4}{|c|}{ PREP. PRESENTACIO } & \multicolumn{3}{|c|}{ PRESENTACION } \\
\hline & CANT & CAL & INIC & ACTIT & \multicolumn{2}{|c|}{ PARTICIPACION } & ACTT & \multirow[t]{2}{*}{ PUNCT/ASID } & \multirow[t]{2}{*}{ CANT } & \multirow[t]{2}{*}{$\mathrm{CAL}$} & \multirow[t]{2}{*}{ INIC } & \multirow{2}{*}{$A C T T$} & \multirow{2}{*}{\begin{tabular}{c|} 
TIEMPO \\
PREV
\end{tabular}} & \multirow{2}{*}{\begin{tabular}{l|} 
TIEMPO \\
PREGS
\end{tabular}} & \multirow{2}{*}{ INFORME } \\
\hline & & & & & CANT & $\mathrm{CAL}$ & & & & & & & & & \\
\hline 1 & $B$ & $B$ & B & $B$ & MB & MB & $B$ & $\mathrm{R}$ & MB & $B$ & B & B & MB & $\mathrm{B}$ & MB \\
\hline 2 & 1 & & 1 & 1 & $\mathrm{~S}$ & $\mathrm{~S}$ & $\mathrm{~S}$ & B & $\mathrm{B}$ & $\mathrm{B}$ & B & B & $\mathrm{B}$ & $*$ & 1 \\
\hline 3 & MB & MB & $R$ & $B$ & B & B & $B$ & $B$ & MB & MB & $B$ & B & $B$ & B & $\mathrm{R}$ \\
\hline 4 & 1 & 1 & 1 & I & 1 & 1 & $\mathrm{I}$ & S & 1 & 1 & 1 & 1 & $*$ & $*$ & 1 \\
\hline 5 & $\mathrm{R}$ & B & $\mathrm{R}$ & B & $\mathrm{R}$ & $\mathrm{R}$ & B & B & $\mathrm{R}$ & $\mathrm{R}$ & B & B & $*$ & $*$ & 1 \\
\hline 6 & MB & MB & B & B & $\mathrm{R}$ & B & B & B & MB & MB & B & B & B & $*$ & B \\
\hline 7 & B & $B$ & $\mathrm{R}$ & $B$ & B & $\mathrm{B}$ & B & B & B & $\mathrm{B}$ & B & B & * & $*$ & B \\
\hline 8 & B & MB & B & B & B & B & MB & MB & B & B & $R$ & B & $*$ & B & 1 \\
\hline 9 & B & B & $\mathrm{R}$ & B & $\mathrm{R}$ & $\mathrm{R}$ & B & B & $\mathrm{R}$ & $\mathrm{R}$ & B & B & * & $*$ & MB \\
\hline 10 & B & B & $\mathrm{R}$ & B & B & MB & MB & MB & $R$ & $R$ & B & B & $*$ & $*$ & MB \\
\hline 11 & B & $R$ & B & B & MB & B & B & B & B & B & $\mathrm{R}$ & B & B & B & MB \\
\hline 12 & B & B & $\mathrm{R}$ & B & $\mathrm{R}$ & B & B & B & $\mathrm{R}$ & $\mathrm{R}$ & $\mathrm{R}$ & B & ${ }^{*}$ & $\star$ & $\mathrm{R}$ \\
\hline 13 & MB & B & B & B & B & B & B & B & B & B & B & B & MB & MB & B \\
\hline 14 & MB & MB & MB & B & B & MB & B & B & B & B & B & B & ${ }^{*}$ & $\bullet$ & $\mathrm{R}$ \\
\hline 15 & B & B & R & B & $R$ & B & B & B & $R$ & $\mathrm{R}$ & $\mathrm{R}$ & B & * & * & I \\
\hline 16 & $B$ & MB & MB & B & B & MB & B & MB & B & B & B & B & * & B & MB \\
\hline
\end{tabular}

Estas valoraciones se presentaron a los estudiantes y en general fueron aceptadas sin problemas. 


\section{Informe sobre la $1^{a}$ valoración}

La valoración del rendimiento para el periodo hasta el 17 de noviembre se presenta en la Tabla 2.

En el caso del alumno 4, las calificaciones insuficientes, provocaron una reclamación al profesor. Problemas de coordinación con el subgrupo que le correspondía y otras causas externas fueron los motivos de dicha reclamación, que intentaron solucionarse de inmediato. De otro modo dichos problemas no hubieran sido detectados. Otra reclamación de carácter menor provino del alumno 5, al cual no se había calificado por carecer los evaluadores de referencias relativas a su trabajo individual. Se rectificó después de escuchar al alumno y verificar el trabajo realizado. Estas valoraciones negativas provocaron preocupación en los demás integrantes del grupo y en el profesor. En el caso del alumno 4, se buscó que su participación en las tareas asignadas fuese más activa.

Los resultados de la valoración fueron comentados en clase una semana después de la primera presentación. El método tuvo aceptación y se hicieron propuestas para mejorar aspectos concretos: se propuso que los alumnos hicieran ellos mismos las valoraciones de sus compañeros de grupo. La propuesta fue aceptada en primera instancia pero no se llevó a término en la segunda valoración. Los inconvenientes con los que se encontró el grupo para desarrollarla, se pueden concretar en: i) no se llegó a un consenso al respecto de a quién tenia que valorar cada estudiante. Dado que el trabajo lo realizó independientemente cada sub-grupo y en ocasiones, el trabajo fue de carácter individual, los estudiantes no tenían datos de primera mano para realizar la valoración objetivamente; y ii) Algunos estudiantes se mostraron reacios a valorar a sus compañeros. Por estas razones, se realizó una segunda valoración con los mismos planteamientos que la primera.

\section{Informe sobre la $2^{a}$ valoración}

En la segunda valoración el trabajo estaba ya muy avanzado y los grupos trabajaban separadamente. Las reuniones pasaron a tener una función de consulta de aspectos puntuales del trabajo de cada grupo Los estudiantes intercambiaban información relativa a los aspectos comunes y. en términos generales, el trabajo se realizó con gran fluidez. Esta actitud positiva de los integrantes del grupo en las reuniones semanales hizo innecesaria la valoración del apartado de trabajo en clase. Si se comparan ambas valoraciones, se puede ver que los insuficientes han desaparecido (Tabla 3). Por otro lado, algunos de los subgrupos vieron disminuida su actividad por la misma dinámica del proyecto, lo que motivó valoraciones inferiores a las de la primera valoración (alumnos 9, 13). La mayoría sin embargo, tuvo valoraciones globalmente mejores (alumnos 2, 3, 4, 5, 7, 8, 12, 13, 14, 15).

Llegados a este punto, se consideró oportuno realizar un estudio de las valoraciones que los alumnos de otros grupos de proyectos habían realizado al respecto de la segunda presentación del grupo. Se buscaba con ello obtener una visión externa que aportase elementos constructivos de cara a posteriores presentaciones. Para ello se realizó un análisis de los informes manuscritos por los alumnos de $5^{\circ}$ curso de la asignatura de proyectos. Estos informes, presentados a continuación, fueron comentados por los alumnos de PLASTEC en clase.

\section{Informe de evaluación de los alumnos}

Este informe se realizó con el objeto de analizar la trayectoria del trabajo del grupo de proyectos respecto a la primera presentación, permitiendo incidir sobre los puntos menos calificados y sobre los ámbitos más criticados. El análisis estadístico se presenta en la tabla 4. La muestra escogida contiene 23 informes de alumnos de $5^{\circ}$ curso de la Escuela Técnica Superior de Ingenieros Industriales de Terrassa e incluye únicamente aquellos que valoraron la presentación del proyecto PLASTEC. Se ha subdividido el estudio atendiendo a los factores que se incluyen en el informe que deben rellenar los alumnos: contenido, dinámica, expresión, medios utilizados y otros comentarios. En el análisis de los comentarios de los estudiantes, se ha intentado ser lo más fiel posible a las opiniones que se reflejan en los informes, manteniendo los adjetivos y matices dados por los estudiantes. 
Tabla 3: Valoraciones de la segunda presentación

\begin{tabular}{|c|c|c|c|c|c|c|c|c|c|c|c|c|c|c|c|}
\hline \multicolumn{2}{|c|}{ GRUPO:92-7 } & & & & & & & \multirow{2}{*}{ PERIODO } & \multirow{2}{*}{\multicolumn{4}{|c|}{$\begin{array}{l}\text { DESDE } 17 / 02 / 1992 \\
\text { HASTA } 18 / 03 / 1993\end{array}$}} & & & \\
\hline & & & & & & & & & & & & & & & \\
\hline \multirow[t]{3}{*}{ ALUMNO } & \multicolumn{4}{|c|}{ TRABAJO INDIVIDUAL } & \multicolumn{4}{|c|}{ TRABAJO EN CLASE } & \multicolumn{4}{|c|}{ PREP. PRESENTACIO } & \multicolumn{3}{|c|}{ PRESENTACION } \\
\hline & CANT & CAL & INIC & ACTIT & PARTI & $\mathrm{AClON}$ & ACTIT & PUNCT/ASID & CANT & CAL & INIC & ACTIT & TIEMPO & TIEMPO & INFORME \\
\hline & & & & & CANT & CAL & & & & & & & PREV & PREGS & \\
\hline 1 & $B$ & MB & MB & MB & $\cdot$ & $*$ & * & * & $B$ & MB & $B$ & $B$ & MB & + & MB \\
\hline 2 & $R$ & $\mathrm{R}$ & $\mathrm{R}$ & $\mathrm{R}$ & * & * & * & * & * & $\cdot$ & * & * & $\bullet$ & * & B \\
\hline 3 & $\bar{R}$ & $\mathrm{R}$ & $\mathrm{R}$ & $\mathrm{R}$ & $*$ & $*$ & $*$ & $*$ & $\bullet$ & $\cdot$ & $\cdot$ & $\cdot$ & $*$ & $*$ & B \\
\hline 4 & $R$ & $\mathrm{R}$ & $R$ & $\mathbf{R}$ & $*$ & $*$ & $*$ & $*$ & * & $*$ & $\bullet$ & $*$ & $*$ & $\cdot$ & MB \\
\hline 5 & B & MB & B & B & $*$ & $*$ & $*$ & $*$ & $*$ & $*$ & $*$ & $*$ & $*$ & $*$ & $\mathrm{R}$ \\
\hline 6 & $\mathrm{R}$ & $\mathrm{R}$ & $\mathrm{R}$ & $\mathrm{R}$ & $*$ & $\star$ & $*$ & $*$ & $*$ & $\star$ & $*$ & $\star$ & $*$ & $*$ & B \\
\hline 7 & B & B & $\mathrm{MB}$ & MB & $*$ & 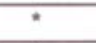 & $*$ & $*$ & B & B & B & B & $*$ & $*$ & B \\
\hline 8 & B & MB & MB & $\mathrm{MB}$ & * & * & * & * & B & MB & MB & MB & * & * & B \\
\hline 9 & B & B & MB & MB & $*$ & $*$ & $*$ & $*$ & MB & MB & MB & MB & $\cdot$ & $*$ & B \\
\hline 10 & MB & MB & MB & MB & $*$ & $*$ & * & $\dot{*}$ & MB & MB & MB & MB & MB & $*$ & MB \\
\hline 11 & $\mathrm{R}$ & B & B & B & $*$ & $*$ & ${ }^{*}$ & $*$ & B & B & B & B & B & $\bar{R}$ & MB \\
\hline 12 & $R$ & $B$ & $R$ & $R$ & $*$ & * & $*$ & $*$ & * & $*$ & 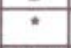 & 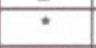 & $*$ & $\cdot$ & B \\
\hline 13 & B & B & B & B & * & * & • & • & B & MB & MB & MB & MB & - & MB \\
\hline 14 & B & B & B & B & $*$ & $*$ & $*$ & $\cdot$ & B & B & B & B & B & $*$ & B \\
\hline 15 & $B$ & B & MB & MB & * & * & * & * & $B$ & B & B & B & * & * & $\mathrm{B}$ \\
\hline 16 & MB & MB & MB & MB & $*$ & ${ }^{*}$ & $*$ & $*$ & B & B & B & B & B & $*$ & MB \\
\hline
\end{tabular}

La puntuación del Contenido del proyecto fue muy favorable. Sobre 23 personas que opinaron sobre este punto. 17 lo hacen favorablemente, un $94.4 \%$ respecto a los que se han manifestado. Una sola persona de las 18, opinó que el contenido era "pobre". Se han incluido dos columnas, una sobre alta precisión y detalles, que puede tener un factor positivo (+) o negativo (-), y la otra sobre falta de detalles o de precisión, con un factor siempre negativo (-). La explicación sobre el "Lay-out" fue valorada como positiva en 4 informes (4+). Por otro lado, en 3 de ellos se consideró que esta era 'demasiado concisa' (3-). Las estructuras fueron 'poco explicadas' (2-) para dos personas. La instalación eléctrica obtuvo la misma puntuación de 3 valorándola tanto positiva como negativamente en la misma proporción. La planificación del trabajo se valoró como "demasiado precisa" (2-) en dos casos, contrastando con un voto a favor (1+). La gran atención prestada a la tabiquería, y el carácter exhaustivo dedicado en la presentación a este tema, ha proporcionado puntos positivos (2+) en dos informes.

A continuación se enumeran los puntos que motivaron comentarios explícitos por parte de los alumnos de otros grupos. En la sesión de trabajo del grupo PLASTEC posterior a la segunda presentación se incidió en cada uno de ellos y se analizó por parte de todo el grupo. Los puntos a mejorar eran: i) Seguridad (1-). Construcción y estructura (4-), ii) Planos (1-). Se deberían mostrar más planos realizados, iii) Taller (1). Explicar más a fondo en que va a consistir el taller y tipos de máquinas disponibles, iv) Aulas y laboratorios (1-), vibraciones y puente grúa (2-).

La relación de puntos a mejorar debe contrastarse con el número de personas que opinan sobre ellos. Solo una o máxime cuatro son las personas que han incidido en su importancia sobre un total de 23 , lo que representa menos que un $17 \%$ del total. Siguiendo con el análisis de los factores a valorar, la Dinámica ha sido valorada como 'buena, clara y ágil' en un $73.7 \%$ relativo a los votantes. La exposición se ha valorado como 'demasiado larga' en un 39.1\% sobre el total, o un $81.8 \%$ sobre los que han opinado sobre dicho punto. La Expresión en la explicación se ha valorado muy favorablemente. Las 14 personas que han opinado han dado una valoración alta. Respecto al apartado de Medios, en todos los informes estudiados se recogen opiniones favorables, pero el rendimiento podría haberse mejorado si se hubiera incidido en factores como: mala visión del proyector de vídeo por excesiva luz. (Atribuido también a una falta de previsión), y por problemas en la proyección de la imagen. Otros comentarios fueron: a) Demasiada gente exponiendo, valorado tanto negativa como positivamente; b) Existían faltas ortográficas o palabras substituibles como "Lay-out", dando como alternativa "distribución en planta".c) Bajo rendimiento de los medios técnicos empleados; y d) Se pedía plasmar datos técnicos en transparencias para que se pudiera comprender mejor el cálculo. 


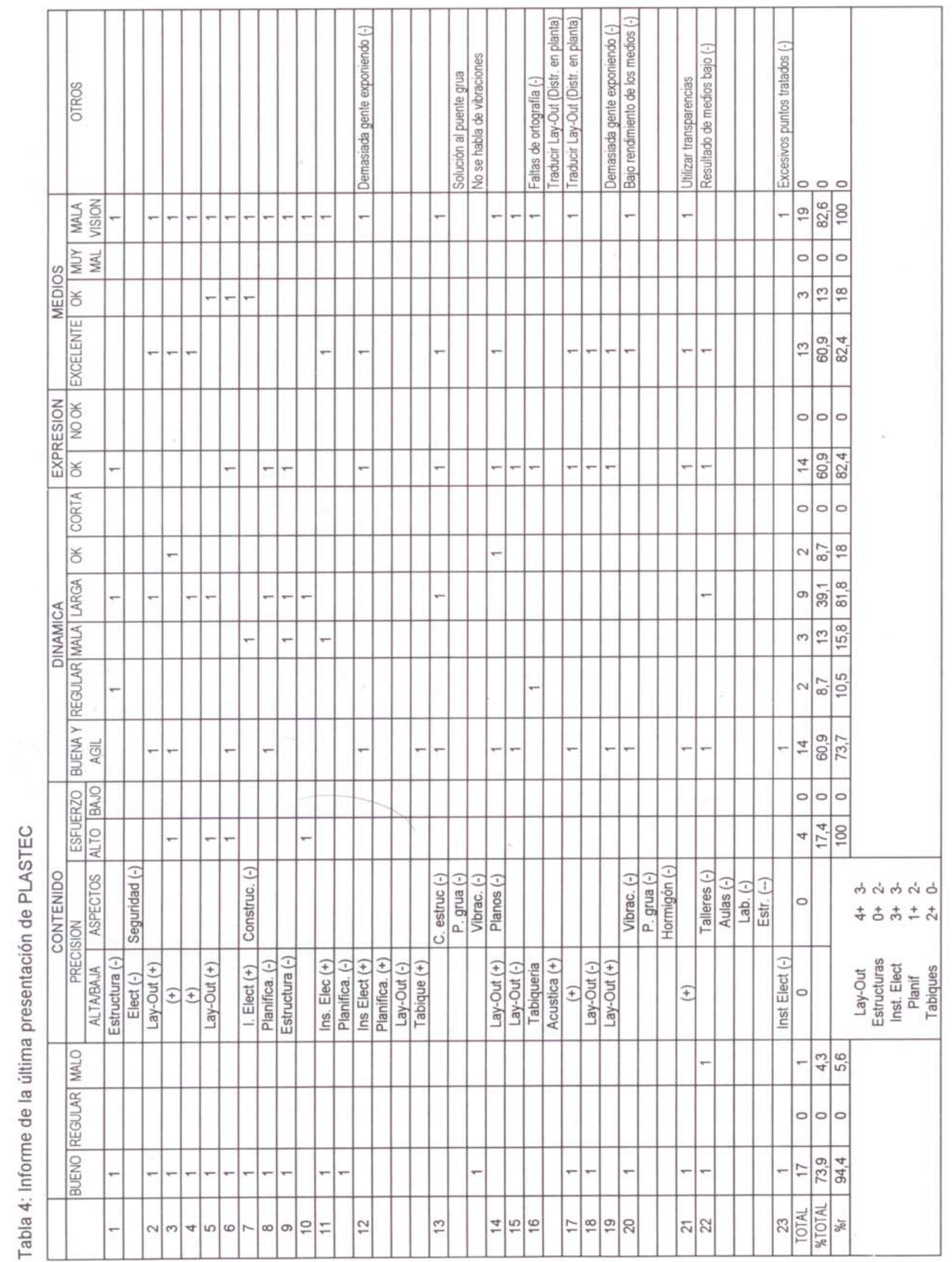

\section{CONCLUSIONES}

La influencia de las valoraciones en el grupo de alumnos de proyectos no se puede considerar en ningún caso despreciable. Algunos de los aspectos mas relevantes que se pueden extraer de las valoraciones presentadas son: 
i) Al proporcionar al alumno la posibilidad de conocer de manera clara y concreta aquellos aspectos de su trabajo que van a ser valorados a lo largo del curso y que constituirán una parte importante de la valoración final, éste puede adaptar su trabajo a los objetivos educativos del curso, sabiendo en todo momento lo que se espera de él.

ii) El efecto de reconocimiento del trabajo bien hecho a la vista de las propias calificaciones es un hecho positivo constatable. Esto lleva consigo una mayor identificación con las tareas del grupo, mejorándose así el ambiente de trabajo y la calidad global del proyecto.

iii) En el caso de calificaciones negativas en una de las valoraciones, el clima de cohesión del grupo, con objetivos claros y consensuados, facilita la reintegración al mismo del alumno con bajo rendimiento y su incorporación a tiempo al ritmo de trabajo de sus compañeros.

iv) El efecto de motivación sobre el trabajo individual, conlleva una mejora del mismo y una predisposición mucho mas positiva por aprender te mas nuevos o profundizar en temas ya conocidos.

v) La valoración de aspectos como la participación en las sesiones de trabajo, la iniciativa o la actitud, fomentan la participación de los estudiantes, la generación de ideas novedosas o el seguimiento de aspectos paralelos que de otro modo no serian tratados. La importancia que se dio a las intervenciones en las sesiones de grupo, tanto cualitativa como cuantitativamente, en la primera valoración, provoco un cambio en la actitud de los alumnos, incrementándose la participación.

vi) La adecuada frecuencia con que se realizaron las valoraciones y el seguimiento individualizado, permitió evitar a tiempo los suspensos a final de curso.

vii) En general, una valoración consensuada y con criterios claros y justos, fomenta un clima de participación muy positivo y una cultura organizativa donde los intereses del grupo priman sobre los individuales, llegando de algún modo a confundirse.

\section{REFERENCIAS}

Anguera, M.T.; "Posibilidades de la metodología cualitativa vs. Cuantitativa", Revista I6 de Investigación Educativa (3,2), 127-145 (1985).

Apple, M.W. y N.R. King; "¿Que enseñan en Escuelas? En J. Gimeno y A. Pérez (Co). "La enseñanza, su teoría y su practica", Madrid, Akal Universitaria (1983).

Beard, R.; "Pedagogía y Didáctica de la Enseñanza Universitaria", Ed. Oikos-Tau, Barcelona (1974).

Bleger, J.; "Grupos operativos en la Enseñanza", Buenos Aires: Nueva Visión (1980).

Bogumil, F.T.; "Proyecto Docente", Dpto. de Proyectos de Ingeniería, Barcelona (1992).

Crum, L.; "Análisis de Valor", P.W.N., Warszawa (1978).

Huse, E.F y J.L. Bowditch; "El Comportamiento Humano en la organización", Deusto, Bilbao (1989).

Nisbet, J. y J. Shuckmiry; "Estrategias de aprendizaje" Madrid, Anaya (1987).

Olszewski, E.; "Disciplina de la Enseñanza", Zycie Szkoly Wyzszej, Warszawa (1975). 